\title{
Kalman filter and a fuzzy logic processor for series arcing fault detection in a home electrical network
}

\author{
Edwin Calderon-Mendoza ${ }^{1}$, Patrick Schweitzer, Serge Weber \\ University of Lorraine, Institut Jean Lamour (IJL), UMR7198, Nancy, F-54000, France
}

\begin{abstract}
This paper presents a method for detecting series arcing faults in AC home electrical networks. The proposed algorithm is based on both a Kalman filter, used for identifying fault symptoms and a decision block, which confirms the presence of a series arc fault to activate a tripping signal. The current measured at one end of the power line is estimated using a model of two steady-state variables (X1 and X2). Firstly, residuals and the third order difference of state X2 are used as input parameters of a Fuzzy logic processor for detecting fault symptoms. Secondly, the fault symptoms are processed by a detection logic block, which confirms the presence of an electrical arcing fault.

The algorithm is tested on a variety of loads in single or masking load configurations chosen accordingly to the requirements of the UL 1699 and IEC 62606 standards. The algorithm is also tested in the steady state or at load start (transient state). This method's performance is studied and discussed in the final part. Experimental results show that the method we propose can detect arcing faults efficiently, avoiding false tripping, whilst taking into account a high degree of diagnosis accuracy and average detection time.
\end{abstract}

Keywords: AC series arcing fault, Fuzzy logic processor, Kalman filter, adaptive threshold.

\section{Introduction}

Arcing fault detection in domestic networks has been an important subject for industrials and researchers for many years. In the USA, the safety standard UL 1699 dating from 1999, includes the requirements for arc fault interrupters (AFCIs) whilst in Europe, standard IEC 62606 is much more recent and has been at the forefront of the development of protective electric arc equipment for home use $(50 \mathrm{~Hz}$ operating frequency and $230 \mathrm{~V}$ supply voltage). Protection devices are mainly based on the power line current and are inserted at the level of the general supply source in dwelling units. The challenge for fault detection algorithms is to work effectively with different circuit configurations where series arcing faults are difficult to identify. In this work, we focus on arc fault situations in presence of masking loads, whilst also taking into consideration the transient effect of starting loads, the EMI filter configuration and disturbing appliances.

The first difficulty in the development of AFCI lies in the choice of one or more optimal criteria. Different arcing fault detection approaches have been proposed in the literature, with spectrum analysis being the detection method most often used. Spectral analyze of the line current focusing on third and fifth harmonics [1-5] is performed in a variety of bands ranging from low frequencies up to $20 \mathrm{kHz}$. In [6] a fault detection algorithm based on the energy density spectrum computation

\footnotetext{
Email address:

edwin-milton.calderon-mendoza@univ-lorraine.fr (Edwin

Calderon-Mendoza)
}

uses an existing structure of a shunt active power filter. The approach presented in [7] analyzes the power spectral density of electromagnetic radiations.

Methods based on the temporal evolution of the current signal use the crest factor [8], inter-period correlations of current [9] or algebraic derivative of the line current [10].

Time-frequency methods are able to analyze non-stationary transient signals produced by arcing faults using essentially the Wavelet packet transform [11-14] and recently the HilbertHuang transform [15]. The performance of these methods is strongly influenced by the sampling frequency, the level of signal decomposition and type of load connected to the power network [16].

In this study, the detection method based on the Kalman Filter has the major advantage of allowing regular temporal estimation through an online digital processing structure, unlike the other methods proposed in the literature which perform detection over a predefined sliding time window $[1-13,15,16]$.

The Kalman filter and the extended kalman filter (EKF) are widely used in fault diagnosis [17-19] and navigation systems [20]. The Kalman filter estimates instantaneous states from noisy (measurement) data recursively. Residuals generation or noise estimation through the variance of the Gaussian distribution [21] is used to identify the presence of a fault.

The use of Kalman filters for protection relays on high power systems developed in the 1980 s by [22], provides state space models for voltages and currents. The work outlined in [23] associates an EKF and a support vector machine (SVM) to detect a parallel arcing fault. [24] presents a method based on two Kalman filters with different dynamics which detect incipient 
faults. This method is able to discriminate arcing faults from switching actions and load changes in an underground cable.

In [25], the Kalman filter is used to calculate eigenvalues which are compared to a predefined reference value (threshold) in order to identify the presence of an arc fault. However, this method was tested in stationary operating mode using mainly resistive household appliances as loads, with no inclusion of motor loads.

An important part of the detection algorithm lies in the decision block which must operate reliably to confirm the presence of an arcing fault, respecting detection times imposed by standards UL 1699 and IEC 62606. Statistical techniques used for the selection of static thresholds presented in [26] are not sufficient to develop robust algorithms capable of avoiding false activation on circuit breakers. Another solution is to use a neural network or an SVM [27-29]. However, they require long learning stages and are difficult to implement in a conventional electronic circuit board. In order to reconcile simplicity and efficiency, the strategy adopted in this work relies on an adaptive thresholding logic. The main part of the decision making is constituted by a Fuzzy logic processor largely used in recent years in fault diagnosis in photovoltaic and robotic systems [30-33] .

The major contribution of this article is the development of an arcing fault algorithm which is designed to reliably detect arcing fault events in stationary and transient operating modes in household appliances. Further, the algorithm incorporates more efficient fault detection and the prevention of nuisance tripping since it is is capable of distinguish arcing faults from load variations in the presence of masking configurations and disturbing appliances.

The paper is organized as follow. Section II gives a functional description of the algorithm put forward for detecting series arcing fault. Section III describes the experimental platform used for series arcing fault tests. Experiments are also carried out to find the frequency at which the Kalman filter can operate optimally. Section IV is dedicated to the decision part. Section $\mathrm{V}$ is concerned with the validation of the algorithm thus developed. Finally section VI concludes this paper and discusses the future scope.

\section{Proposed method}

The block diagram of the algorithm represented in Fig. 1, consists mainly of a Kalman filter block and a decision part based on Fuzzy logic.

The kalman filter input is the current measured at one end of the power line (close to the source). The current signal is estimated by using the calculation of two-state variables X1 and X2 obtained from state equations. Residuals (Res) obtained from the subtraction between the measured current and its estimation and also the third order difference of state X2 are used by the decision block. This block consists of a Fuzzy logic processor responsible for generating fault symptoms which are processed by a detection logic block to confirm the presence of an arcing fault. Finally this block sends a tripping signal (TS) to activate a controlled switch in order to disconnect the power source from the power line.

\subsection{Kalman filtering}

The mathematical model of a dynamic system can be described using a state-space representation in the following form:

$$
\begin{aligned}
X(n+1) & =A * X(n)+W(n) \\
Y(n) & =C * X(n)+V(n)
\end{aligned}
$$

where $X(n)$ is the process state vector, $A$ is the transition matrix, $W(n)$ is the process noise matrix, $Y(n)$ is the measured output, $C$ is the output matrix and $V(n)$ is the measurement noise matrix.

The process noise $W(n)$ is directly related to a series arcing fault in the power line whose dynamic is unknown. The measurement noise matrix $V(n)$ mainly relates to the noise introduced by the current sensor. Both of them are assumed to be non correlated and zero-mean white noise processes.

Covariance parameters obtained from $W(n)$ and $V(n)$ are used in the designing of a Kalman filter. These parameters represent a trade-off between a faster filter response and better attenuation in the filter estimates. The symmetric covariance matrices are defined by $Q=E\left[W^{T} W\right]$ and $R=E\left[V^{T} V\right]$.

The Kalman filter estimates a signal in the discrete domain and filters noises. The current signal in normal circuit operation can be represented by two phasors: the first with initial phase of 0 degree and the second with a 90 degree shift.

$$
i(n)=X 1 * \cos w_{o} t-X 2 * \sin w_{o} t
$$

Where X1 and X2 are independent, zero mean, Gaussian random variables and represent the real and imaginary parts.The optimal estimator Kalman filter used in this work is represented by a two-state model developed by Girgis [22].

$$
\begin{aligned}
& {\left[\begin{array}{l}
X 1(n+1) \\
X 2(n+1)
\end{array}\right]=\left[\begin{array}{ll}
1 & 0 \\
0 & 1
\end{array}\right]\left[\begin{array}{l}
X 1(n) \\
X 2(n)
\end{array}\right]+[W(n)]} \\
& {[i(n)]=\left[\cos w_{o} n \Delta t-\sin w_{o} n \Delta t\right]\left[\begin{array}{l}
X 1(n) \\
X 2(n)
\end{array}\right]+[V(n)]}
\end{aligned}
$$

Equations (4) and (5) represent the state and measurement equations respectively. The recursive estimation of X1 and X2, in the presence of measurement noise, begins with their initialization using initial values, and a previous assignment of the error covariance matrix $P(n-1)=Q$. The prediction mechanism of a Kalman filter estimates the predicted state, the error covariance matrix $(\mathrm{P}(\mathrm{n}))$, the Kalman gain $(\mathrm{Kg})$ using Equations (6), (7) and (8).

$$
\begin{aligned}
\hat{X}(n) & =A \hat{X}(n-1) \\
P(n) & =A P(n-1) A^{T}+Q \\
K g & =P(n) C^{T} \operatorname{inv}\left(C P C^{T}+R\right)
\end{aligned}
$$




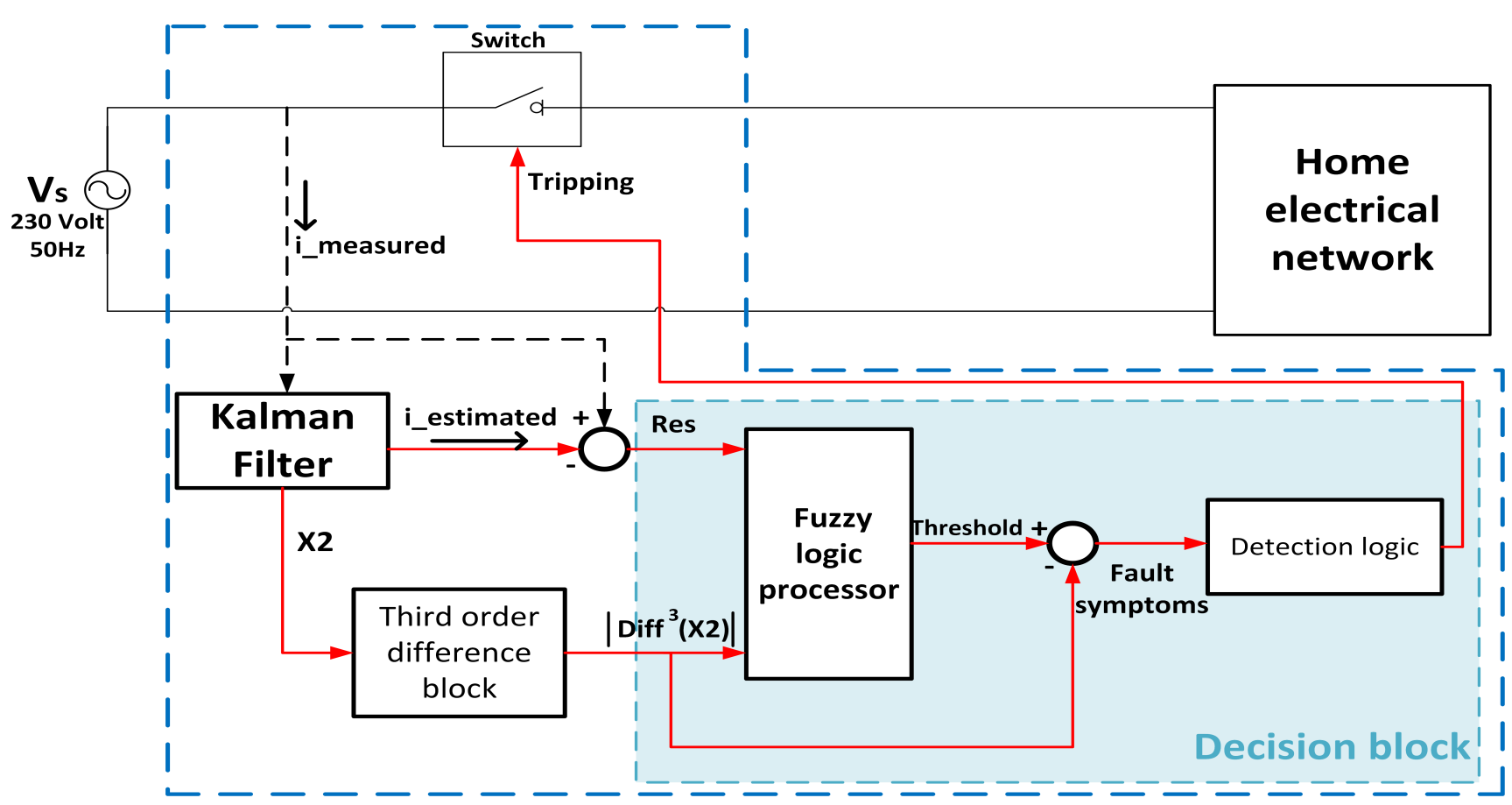

Figure 1: Scheme proposed for AC series arcing fault detection.

$$
\begin{aligned}
\text { inno } & =i(n)-C \hat{X}(n) \\
\hat{X}(n+1) & =\hat{X}(n)+K g * \text { inno } \\
{[P(n+1)] } & =\left[\begin{array}{ll}
1 & 0 \\
0 & 1
\end{array}\right] *[P(n)]-[K g * C * P(n)] \\
i_{\text {estimated }} & =C \hat{X}(n+1)
\end{aligned}
$$

The correction mechanism employs Equations (9), (10) and (11) to calculate the innovation (inno), the corrected state vector and the error covariance matrix. Finally, the estimated output signal is obtained from Equation (12).

For stability analysis purposes, the Kalman filter dynamic can be also expressed substituting Equation (6) in (10).

$$
\hat{X}(n+1)=A \hat{X}(n)+K g *(i(n)-C \hat{X}(n))
$$

Equation (13) represents the dynamic of a predictor type Kalman filter. The error is defined as: $e(n)=x(n)-\hat{X}(n)$ and its discrete-time derivative as: $e(n+1)=x(n+1)-\hat{X}(n+1)$. Substituting Equations (13) and (4) in this last expression allow us to obtain Equation (14).

$$
e(n+1)=(A-K g * C) e(n)
$$

The Kalman filter is asymptotically stable if the eigenvalues of the matrix: $A-K g * C$ are alway inside the unity circle.
Taking into account this constrain, the selection of $Q$ and $R$ must guarantee the stability of the system but also allows a fast dynamic response so that the estimated signal can swiftly track the real signal essentially in normal operating conditions.

The Kalman filter tuning is based on the procedure presented in [34]. We initialize $Q$ and $R$ with identity matrices which keep the filter stability. Then, adjusting tests of matrix $Q$ are done maintaining $R$ constant. The objective of this procedure is to obtain few noise on estimated state values when a fault event is produced. This condition can be achieved decreasing values of matrix $Q$. Thus, after some tests in MATLAB, the optimal matrices are:

$$
Q=\left[\begin{array}{cc}
0.001 & 0 \\
0 & 0.001
\end{array}\right] \quad R=\left[\begin{array}{ll}
1 & 0 \\
0 & 1
\end{array}\right]
$$

These matrices values allow a solution asymptotically stable of Equation (14).

\section{Experimental environment}

An experimental platform is used to create a database of current signatures under normal operation (healthy mode) and then in the presence of series arc faults. The database is used to evaluate the performance of the proposed method of detection.

The process of generating a series arcing fault is done using the opening contacts mode between two copper electrodes $(6$ $\mathrm{mm}$ diameters) according to IEC 62606 standard. The arc generator comprises in particular a stationary electrode and a moving electrode for arc ignition. The separation of two electrodes at an appropriate distance causes the formation of an electric arc. Fig. 2 shows the diagram of the generator. 


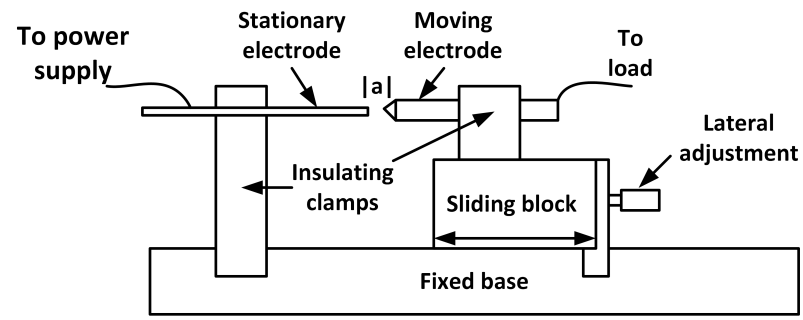

Figure 2: Arc generator by contact breakage.

The experimental setup, represented in Fig. 3, consists of various domestic loads supplied by the European domestic alternating voltage 230 Volts, $50 \mathrm{~Hz}$.

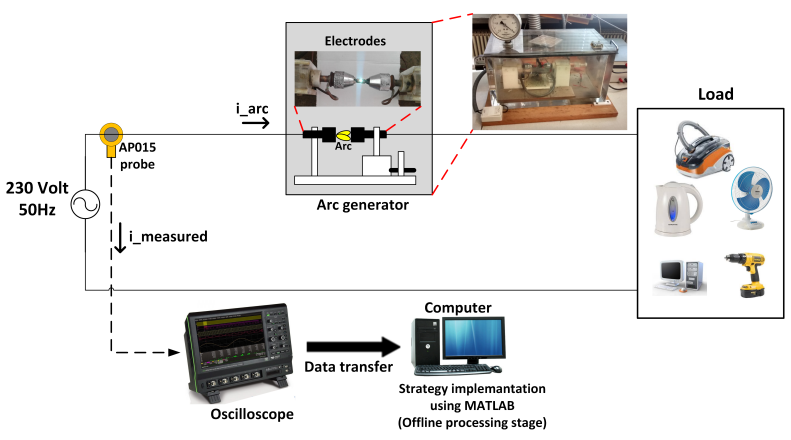

Figure 3: Experimental platform for series arc fault generation.

Measurements are recorded by an oscilloscope Lecroy HDO 6104 which has a deep memory capacity (up to 250 Mpts/Channel) at a sampling rate of $1 \mathrm{MHz}$. Current measurements are made using a Lecroy AP015 current probe $(75 \mathrm{MHz}$ bandwidth). The experiment starts with the normal operating condition (the electrodes are held in contact) in the circuit. An arcing fault is then produced by the electrodes opening. At each zero crossing of the current, the electric arc is switched off and reignited.

The non arcing and arcing fault current waves are recorded by the oscilloscope. The window time for each test is comprised between $1 \mathrm{~s}$ to $2 \mathrm{~s}$ (max. recording of $2 \mathrm{Mpts}$ ). Oscilloscope data is then transferred to a computer. All analysis is done using MATLAB software tool in order to evaluate the performance of the proposed method of detection.

\subsection{Series arc fault tests using typical domestic loads}

Table 1 shows the characteristics of the main linear and nonlinear household loads connected in the circuit. A database of current signatures is obtained using simple and combined loads configuration. The combination of different loads can be made according to several configurations presented in the standards UL 1699 and IEC 62606.

As fault detection is more difficult to achieve with combined loads (table 1), household appliances are associated in parallel. Other tests are performed according to masking configurations presented in Fig. 4. In masking-type configurations a resistor of $80 \Omega$ generates a masking effect in the circuit. In parallel
Table 1: Loads used in the experimental platform.

\begin{tabular}{|c|c|c|}
\hline Configuration & Appliance & $\begin{array}{l}\text { Power } \\
{[\mathrm{W}]}\end{array}$ \\
\hline \multirow{7}{*}{ Simple } & Kettle & 1200 \\
\hline & Halogen lamp & 500 \\
\hline & Fan & 60 \\
\hline & $\mathrm{PC}$ & 300 \\
\hline & Drill & 400 \\
\hline & Vacuum cleaner 1 & 1000 \\
\hline & Vacuum cleaner 2 & 1600 \\
\hline \multirow{2}{*}{ Parallel appliances } & Halogen lamp // Halogen lamp & 1000 \\
\hline & $\mathrm{PC} / / \mathrm{PC}$ & 600 \\
\hline \multirow{3}{*}{ Masking type 1} & Halogen lamp // R=80ohm & \\
\hline & Vacuum cleaner $2 / / \mathrm{R}=80 \Omega$ & \\
\hline & Drill $/ / \mathrm{R}=80 \Omega$ & \\
\hline \multirow{2}{*}{ Masking type 2} & Vacuum cleaner $2 / / \mathrm{R}=80 \Omega$ & \\
\hline & Drill $/ / \mathrm{R}=80 \Omega$ & \\
\hline \multirow{2}{*}{ Masking type 3} & Vacuum cleaner $2 / / \mathrm{R}=80 \Omega$ & \\
\hline & Drill // $\mathrm{R}=80 \Omega$ & \\
\hline \multirow{3}{*}{ EMI filter 1} & $\mathrm{R}=47 \Omega$ & \\
\hline & Drill & \\
\hline & Vacuum cleaner 2 & \\
\hline \multirow{5}{*}{ Disturbing appliance } & Vacuum cleaner 1 & \\
\hline & PC & \\
\hline & Drill & \\
\hline & fluorescent lamp & \\
\hline & Compressor & \\
\hline
\end{tabular}

appliance configuration, and for the first masking-type configuration, the series arc fault is generated between the wire which links the power supply and the parallel loads. In the second masking-type configuration, the arcing fault is generated in series with the resistor. In the third configuration (Masking-type 3 ), the arcing fault is located in series with the load.

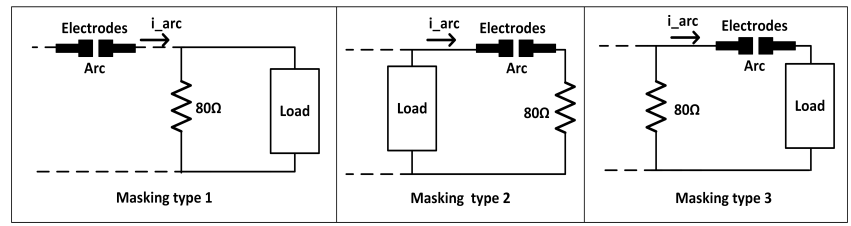

Figure 4: Different masking-type configurations.

Standard IEC 62606 also recommends EMI testing following the experiment represented in Fig. 5. The wire has precise characteristics $\left(\right.$ length $=24 \mathrm{~m}$, section $\left.=2.5 \mathrm{~mm}^{2}\right)$. Two capacitors of $0.22 \mu \mathrm{f}$ are inserted in the circuit.

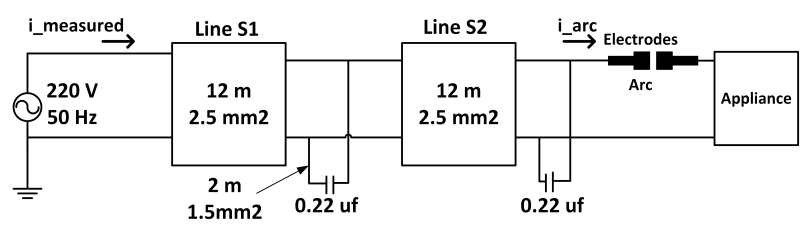

Figure 5: EMI filter.

EMI filter may provide a masking effect on the arcing fault current by preventing the detection of faults with conventional arcing fault circuit breakers.

Another circuit configuration that can disrupt the proper operation of the detector is shown in Fig. 6. A disturbing ap- 


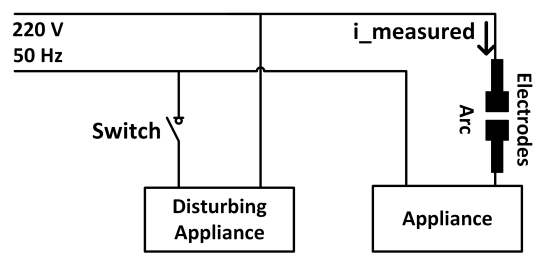

Figure 6: Arcing fault using a disturbing appliance configuration.

pliance is inserted in a branch parallel to the protected load. Variations and starts of disturbing loads can thus be interpreted as faults by the protection circuit. All these tests make it possible to reproduce situations for which the detection of an arc fault is particularly difficult to effect or situations for which a false detection is favored.

\subsection{Selection of the frequency rate}

The use of a Kalman filter firstly requires the selection of a frequency rate at which the filter can operate optimally. For this purpose a simple configuration consisting of a power resistor of $48 \Omega-1000 \mathrm{~W}$ is employed in the experimental platform. $\operatorname{Res}(\mathrm{n})$ is obtained by calculating the difference between a sample current and its estimation using a Kalman filter according to equation (15).

$$
\operatorname{Res}(n)=\left(i_{\text {measured }}(n)-i_{\text {estimated }}(n)\right)^{2}
$$

Three sample rates of $1 \mathrm{kHz}, 100 \mathrm{kHz}$ and $1 \mathrm{MHz}$ were selected. The current signal in normal and then in fault conditions, as well the generation of Res, are displayed in Fig. 7 to show the influence of the frequency rate on the dynamic of the filter. The mode of operation is as follows: when an arcing fault is produced, the dynamic of the filter cannot track all shape changes presented in the current waveform. Consequently Res amplitudes become significant, indicating the presence of an arcing fault. However, the slow dynamic response of the Kalman filter which operates at $1 \mathrm{kHz}$, generates at the beginning of the estimation, residuals (Res) with high amplitudes $(t<0.2 \mathrm{sec})$ in normal circuit operation. The increase in frequency allows a fast filter response. Therefore, at $100 \mathrm{kHz}$ and also at $1 \mathrm{MHz}$, Res have high amplitudes only in the presence of an arcing fault event. Based on similar dynamic responses at these frequencies, the frequency rate of $100 \mathrm{kHz}$ was selected in order to avoid an unnecessary computational burden for the algorithm implementation in an electronic circuit board (FPGA, DSP, etc).

The presence of an arcing fault can be easily detected from the estimation of state X2 and Res in stationary operating mode. In Fig. 8a, an arcing fault is identifiable using a resistive appliance in a simple configuration. However, when using a vacuum cleaner (Fig. 8b), amplitudes of Res are not significantly affected by an arcing fault. On the other hand, an amplitude drop in state X 2 can be observed at $t=0.5 \mathrm{~s}$ indicating the presence of a fault.

Taking into account the results shown in Fig. 8, a strategy for arcing fault detection based only on Res does not allow for
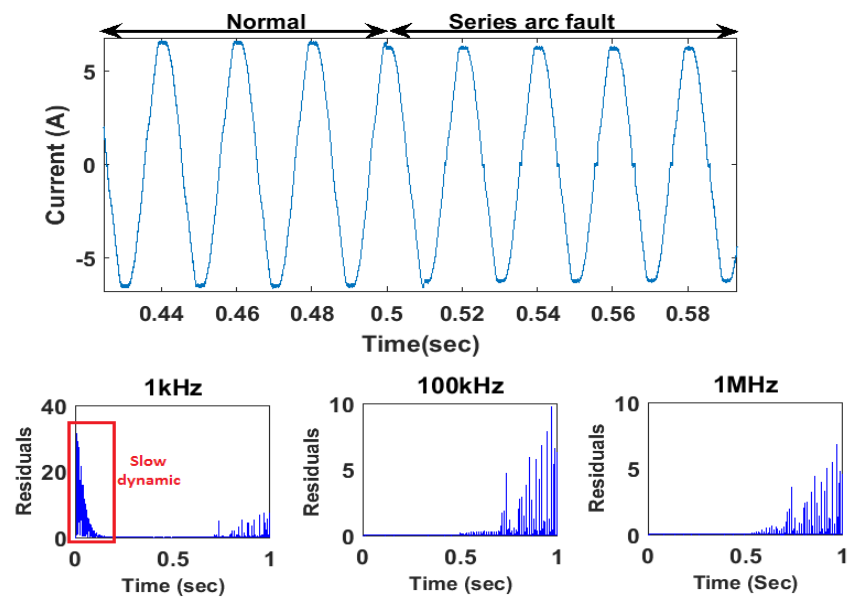

Figure 7: Residuals generation using different frequency rates.
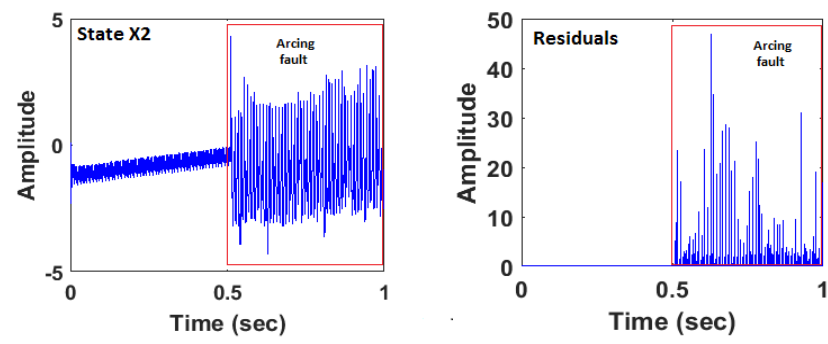

(a) Kettle in stationary operating mode.
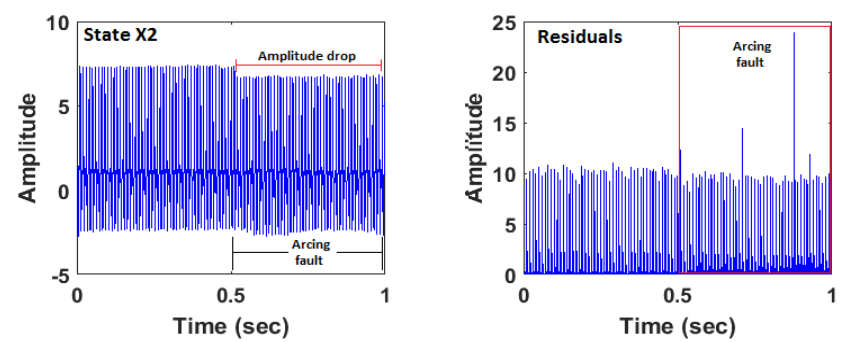

(b) Vacuum cleaner 2 in stationary operating mode.

Figure 8: State X2 and residuals.

effective detection. Thus, Res and state X2 parameters can be combined to detect arcing faults. It can be seen from experiments that the imaginary state $\mathrm{X} 2$ is far more sensitive to the fault presence compared to X1. The association of these two parameters will allow arcing faults to be detected in the presence of motor loads under complex operating conditions (different masking load configurations, transient effects, variable speed and motor torque variations).

Nevertheless, it is preferable to increase the variations in state $\mathrm{X} 2$ in order to obtain strong variations in the signal in presence of arcing faults. In order to effect this, processing based on third order difference of $\mathrm{X} 2\left(\operatorname{diff}^{3}(X 2)\right)$ is carried out and presented in the next subsection.

\subsection{The third order difference}

A derivative type filtering method for arcing fault detection is presented in [35]. According to the authors, this method is based on the third derivative of the arc current which produces 
large logarithms gains preserving the low and high frequency spectral data. However, the derivative function presents the disadvantage of being inherently sensitivity to noise [10]. In order to tackle this problem an approach based on the third order difference is employed. In this regard, the third order difference of a function detects the change of a function instead of computing the rate of change. Algorithms based on the third order difference to detect the core saturation in electrical transformers are presented in $[36,37]$. In these papers the discontinuity of measured currents are identified using the first order difference. The second and the third order differences convert the points of inflection into pulses which are used as fault symptoms.

Based on a similar approach, the third order difference $\operatorname{diff} f^{3}\left(X_{2}\right)$ for arcing fault detection is obtained from the following Equations:

$$
\begin{aligned}
\operatorname{diff}^{1}[X 2(n)] & =X 2(n)-X 2(n-1) \\
\operatorname{diff}^{2}[X 2(n)] & =\operatorname{diff}^{1}[X 2(n)]-\operatorname{diff}^{1}[X 2(n-1)] \\
\operatorname{diff}^{3}[X 2(n)] & =\operatorname{diff}^{2}[X 2(n)]-\operatorname{diff}^{2}[X 2(n-1)]
\end{aligned}
$$

The calculation of $\left|d i f f^{3}(X 2)\right|$ as a function of the state $\mathrm{X} 2$ is performed recursively using the Equation (19).

$$
\left|\operatorname{diff}^{3}(X 2(n))\right|=G *|X 2(n-3)-3 X 2(n-2)+3 X 2(n-1)-X 2(n)|
$$

This equation takes into account a gain factor $(\mathrm{G})$ which will be set experimentally in order to amplify the third order difference coefficients to be used in the generation of fault symptoms. The first coefficient $\left|\operatorname{diff}^{3}(X 2(n=1))\right|$ is calculated using the first four samples of $X 2$ (dependency from previous stored values: $X 2(n-3), X 2(n-2), X 2(n-1)$ and $X 2(n))$.

\section{The decision part}

The decision part plays a key role in the ability of the algorithm to avoid false tripping alarms.

\subsection{Adaptive threshold using a Fuzzy processor}

Based on the Fuzzy approach for threshold adaptation, we present our own topology displayed in Fig. 9. In our system Res and $\left|\operatorname{dif} f^{3}(X 2)\right|$ are used as inputs of a Fuzzy logic processor (Mamdani FIS engine). These parameters deviate from zero even when no fault is present. The deviations in stationary operating mode are due mainly to measurement noises and unknown perturbation inputs. Furthermore, in transient operating mode these deviations vary dynamically and depend on appliance type and circuit configuration. The evaluation of these parameters therefore require a decision making process in order to reduce sensitivity to false alarms. In the adaptive thresholding logic shown in Fig. 9, the Fuzzy logic processor uses Fuzzy rules to sets an optimal threshold $\Delta J\left(\operatorname{Res},\left|\operatorname{diff}^{3}(X 2)\right|\right)$ which is then compared to $\left|d i f f^{3}(X 2)\right|$ coefficients in order to generate fault symptoms.

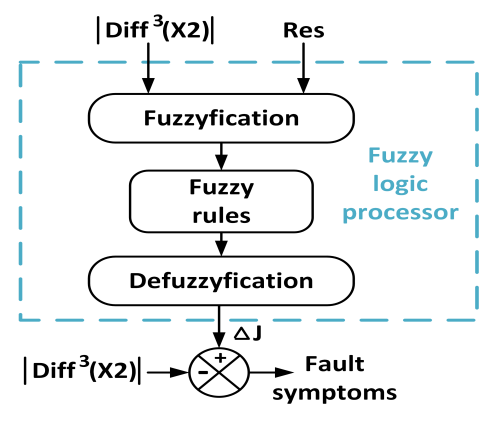

Figure 9: Fuzzy thresholding logic.

$\Delta J$ increases or decreases under different fault scenarios (it includes varying operating conditions and uncertainties). The larger the difference between $\left|\operatorname{diff}^{3}(X 2)\right|$ coefficients and $\Delta J$ the greater the possibility that a fault has occurred.

The implementation of an adaptive thresholding logic includes the following main steps:

1. Heuristic knowledge obtained from the home electrical circuit under different fault scenarios and identification of possible false alarms,

2. fuzzyfication of Res, $\left|\operatorname{dif} f^{3}(X 2)\right|$ and $\Delta J$ by choosing adequate membership functions and,

3. definition of thresholding fuzzy rules.

The heuristic knowledge will be used in the fuzzyfication process and the formulation Fuzzy rules.

\subsection{Fuzzyfication and diagnosis rules}

The mapping of crisp values into a representation by fuzzy sets is required. Thus, the fuzzyfication of Res, $\left|\operatorname{dif} f^{3}(X 2)\right|$ and $\Delta J$ are done through linguistic values. For this purpose, values obtained from experimental tests in stationary operating mode are used as reference. Table 2 displays maximum amplitudes of Res and $\left|d i f f^{3}(X 2)\right|$ coefficients obtained in presence of arcing faults.

Table 2: Reference values.

\begin{tabular}{lll}
\hline Appliance & Max. Res & Max. $\left|\operatorname{diff}_{3}\left(X_{2}\right)\right|$ \\
\hline Kettle & $<25$ & $<1500$ \\
Halogen lamp & $<5$ & $<250$ \\
Fan & $<0.03$ & $<120$ \\
PC & $<1.5$ & $<300$ \\
Drill & $<0.4$ & $<820$ \\
Vacuum cleaner 1 & $<100$ & $<2000$ \\
Vacuum cleaner 2 & $<15$ (constant) & $<1800$ \\
\hline
\end{tabular}

A first analysis show that both Res and $\left|\operatorname{diff} f^{3}(X 2)\right|$ have low amplitudes in absence of arcing faults. Conversely, arcing faults generate high amplitude pulses which depend on the type of household appliance used. In Table 2, the highest amplitude values are obtained for vacuum cleaner 1 and the lowest amplitudes are observed for a fan.

In the case of the vacuum cleaner 2 , Res have similar amplitudes regardless of fault presence. However, $\mid$ dif $f_{3}\left(X_{2}\right) \mid$ have high amplitude pulses when a fault occurs. 
The reference values obtained in Table 2 an the knowledge obtained from currents in transient operating mode (displayed in results) are used in the design of membership functions. The membership functions with amplitudes covering the interval $(0,1)$ are defined by geometric forms that correspond to each linguistic description (low, medium and high). The three groups of customized membership functions are presented in Fig. 10.
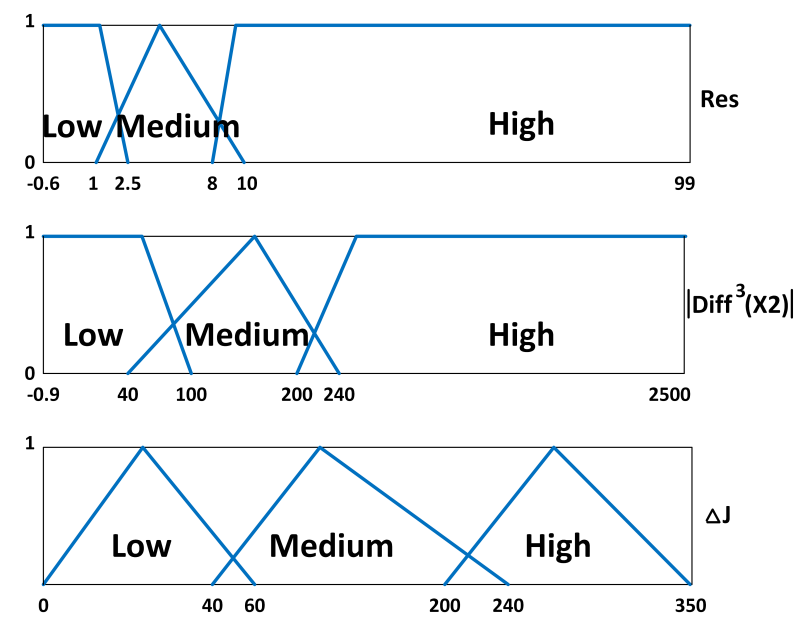

Figure 10: Fuzzy sets.

Six Fuzzy rules found experimentally and listed in Table 3 are capable of adjusting the threshold level efficiently.

Table 3: Fuzzy rules.

\begin{tabular}{ll}
\hline Rule & Rule statement \\
\hline R1 & If Res is Low AND $\left|\operatorname{diff}^{3}\left(X_{2}\right)\right|$ is Low THEN $\Delta J$ is High \\
R2 & If Res is Low AND $\left|\operatorname{diff}^{3}\left(X_{2}\right)\right|$ is Medium THEN $\Delta J$ is Low \\
R3 & If Res is Medium AND $\left|\operatorname{diff}^{3}\left(X_{2}\right)\right|$ is High THEN $\Delta J$ is High \\
R4 & If Res is High AND $\left|\operatorname{diff}^{3}\left(X_{2}\right)\right|$ is Medium THEN $\Delta J$ is High \\
R5 & If Res is Medium AND $\left|\operatorname{diff}^{3}\left(X_{2}\right)\right|$ is Medium THEN $\Delta J$ is Medium \\
R6 & If Res is High AND $\left|\operatorname{diff}^{3}\left(X_{2}\right)\right|$ is High THEN $\Delta J$ is High \\
\hline
\end{tabular}

\subsection{Detection logic}

Anomalous situations unrelated to the presence of a fault can be generated by a high current peak produced when a load is plugged in. This phenomenon of short duration cannot be properly tracked by the Kalman filter. Consequently, Res and $\mid$ dif $f^{3}(X 2) \mid$ have high amplitudes. The thresholding logic can also generate false fault symptoms. The detection logic block shown in Fig. 11 guarantees correct fault detection. This block confirms the presence of an arcing fault and also reduces false tripping as we will see in the next section.

In the process of confirming an arcing fault, the detection logic block starts by transforming the fault symptoms generated by the thresholding logic into binary pulses obtained with a comparator block. Then, a timing window of $\delta \mathrm{ms}$ is then activated by the rising edge of the first binary pulse. An arcing fault is detected when $N$ binary pulses are counted in a timing window. $\delta=60 \mathrm{~ms}$ and $N=7$ are set experimentally in order to get a fast response in the circuit breaker.

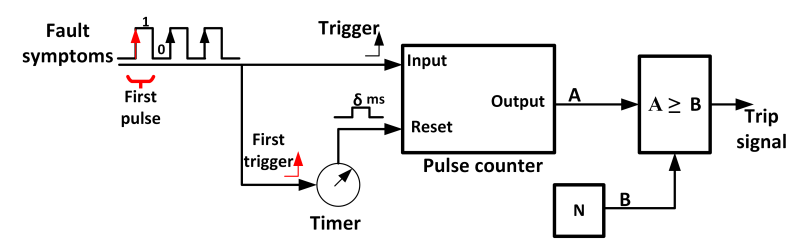

Figure 11: Detection logic.

\section{Results}

The implementation of the schematic representation of the algorithm presented in Fig. 1 was done in a computer using MATLAB code. In order to evaluate and validate the diagnostic accuracy of the detection algorithm, the database obtained from different experimental tests is analyzed through different cases presented as follow.

\subsection{Case 1: stationary operating mode}

The stationary operating mode requires fixed speed and no power variations of household appliances under the presence of arcing fault events. Fig. 11 displays measured current signals (blue color) and their estimations using a Kalman filter (red color) for appliances in simple configuration listed in table 1 taking into account different powers levels. Res, $\left|d i f f^{3}(X 2)\right|$ and fault symptoms with tripping signals obtained in the decision part for arcing fault detection are also displayed.

The algorithm performs correct fault detections and avoids false tripping. The arcing fault is detected after $20 \mathrm{~ms}$ for a PC, a drill and vacuum cleaner 2. In the case of the Kettle and halogen lamp, the arcing fault is detected after $40 \mathrm{~ms}$ and $60 \mathrm{~ms}$ respectively. The arcing fault in a fan with an operating current of $0.5 \mathrm{~A}$ is detected after almost $200 \mathrm{~ms}$.

All the results obtained in stationary operating mode satisfy the triggering time imposed by the standard IEC 62606 considering a supply voltage of $230 \mathrm{~V}$.

\subsection{Case 2: transient operating mode}

\subsubsection{Sub-case 1}

In Fig. 12, the transient operating mode of the current for vacuum cleaner 2 (simple configuration) is shown. The presence of transient current in the initial time (blue color) cannot be properly tracked by the Kalman filter (red color). Consequently, in section A, high variations of Res amplitudes are created. In this case, a decision based only on Res generation could produce false fault symptoms. In section B the transient condition of the current is maintained with the strong and abrupt speed variation of the vacuum cleaner. Also in this section, an arcing fault is generated. Res and $\left|\operatorname{diff}^{3}(X 2)\right|$ coefficients have high amplitudes. The influence of speed variation continues in section $\mathrm{C}$. The residuals are still strongly persistent independent of the arcing fault phenomenon (no arcing fault in section $\mathrm{C}$ ). However, $\left|\operatorname{diff}^{3}(X 2)\right|$ coefficients have low amplitudes, which correspond to a healthy operating mode on the circuit. In sections $\mathrm{D}$ and $\mathrm{E}$, the measured current reaches the stationary state and a slight distortion of the current waveforms is observed with 

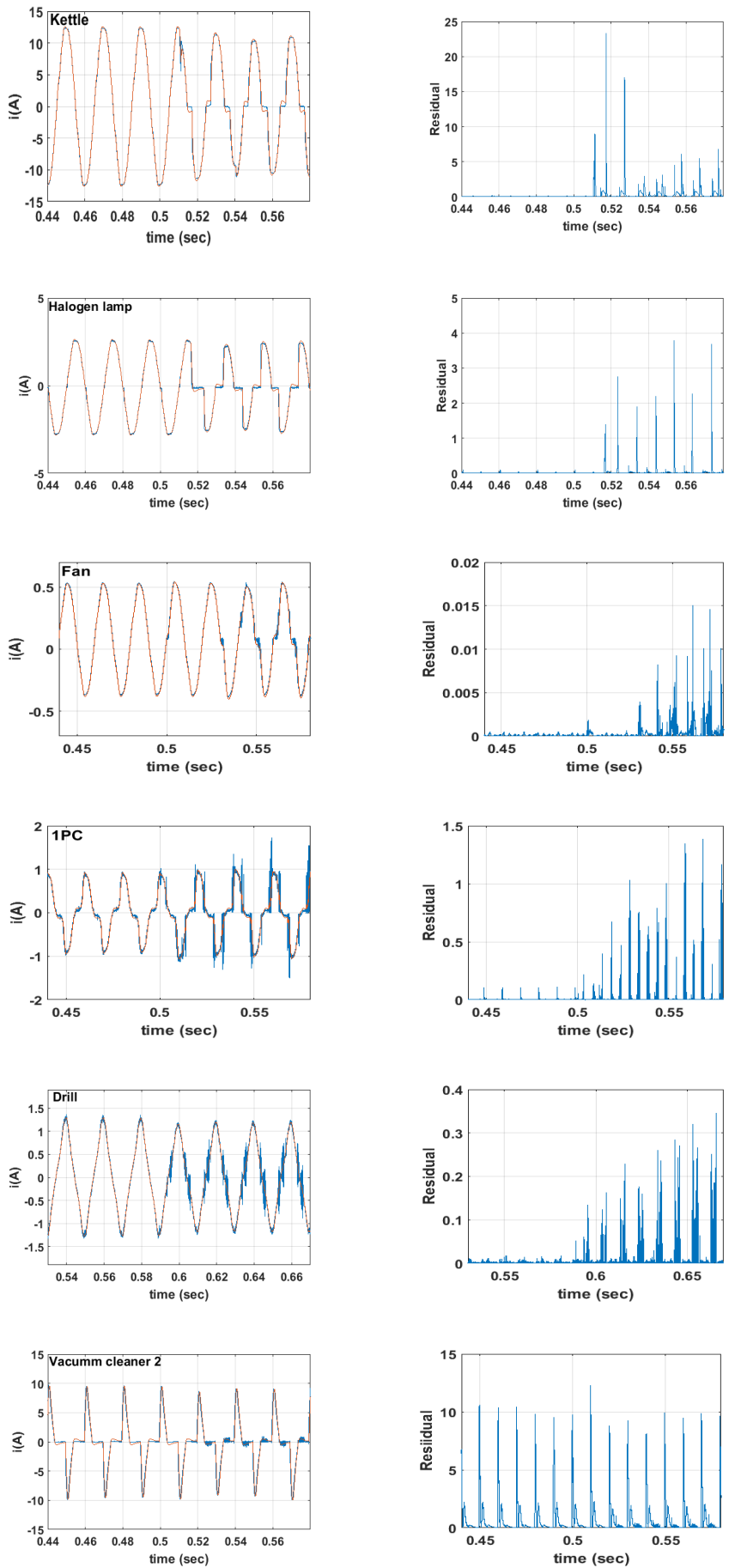

(a) Test signal.
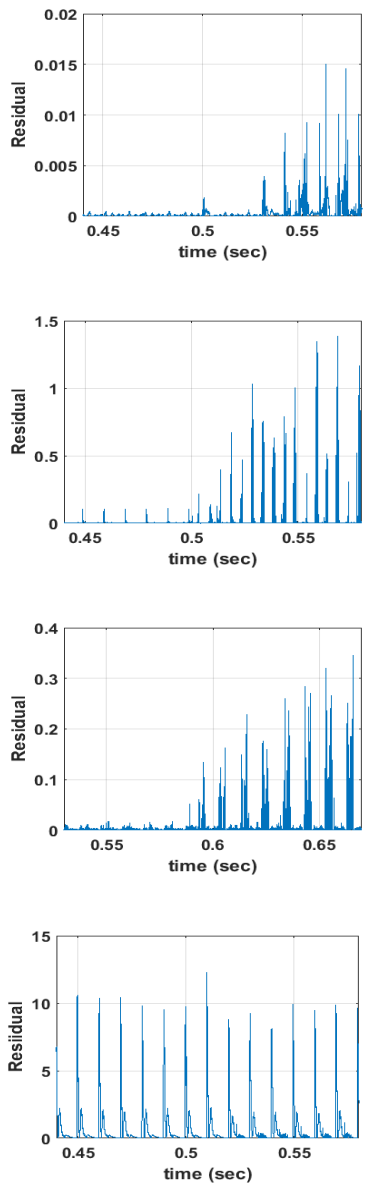

(b) Residuals.
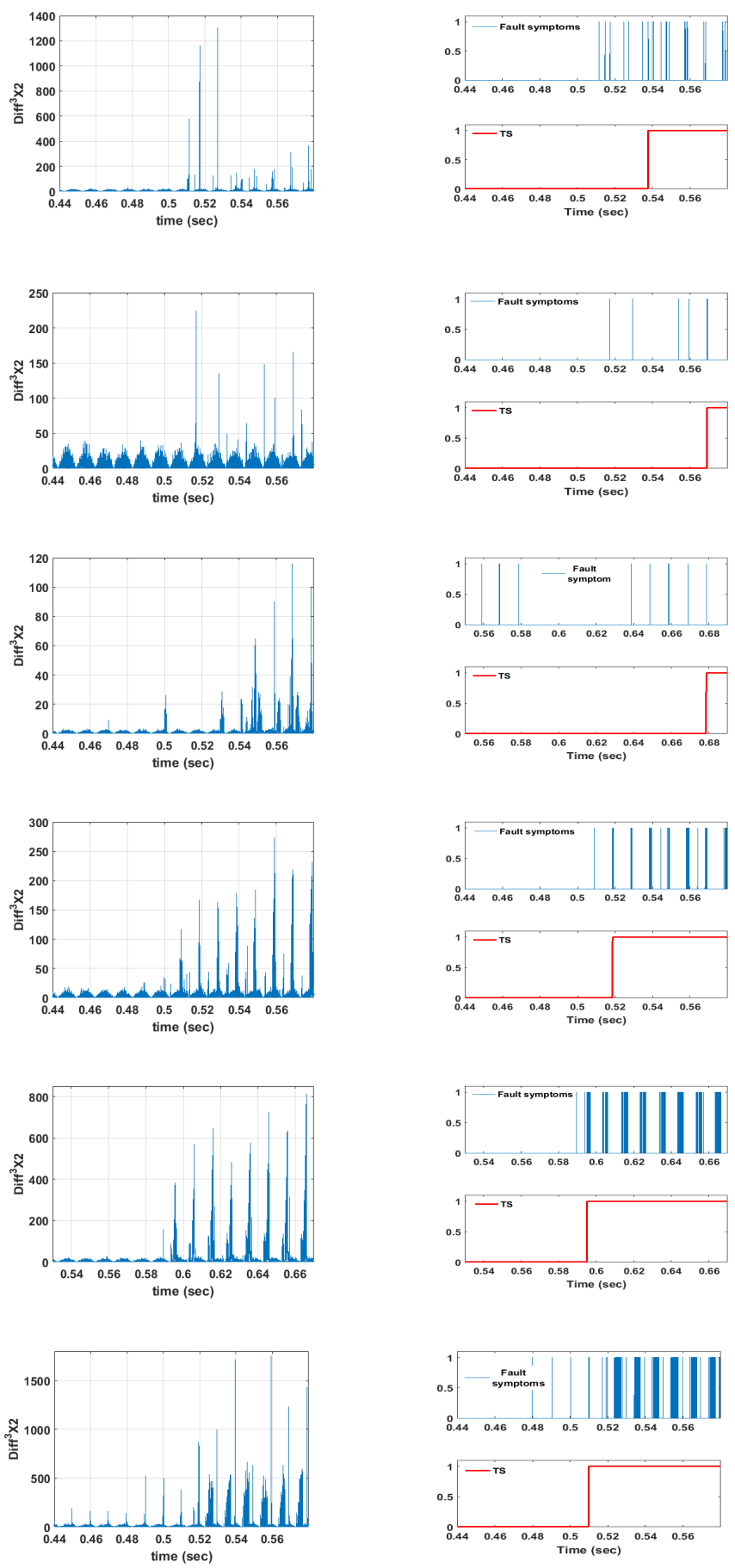

(c) $\left|\operatorname{diff}^{3}(X 2)\right|$.

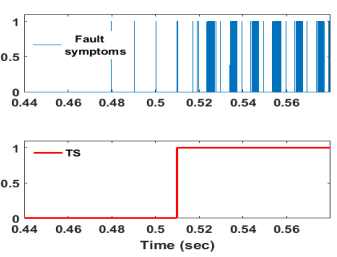

(d) Decision part.

Figure 11: Fault detection in stationary operating mode.

respect to the transient waveforms. In section $\mathrm{D}$, the arcing fault is reignited. However, the fault phenomenon has no influence in Res which keeps low amplitudes. In contrast, $\left|\operatorname{dif} f^{3}(X 2)\right|$ coefficients have persistently high amplitudes. Finally, in section $\mathrm{E}$ the current is reaches a steady state and low amplitudes of residuals and $\mid$ dif $f^{3}(X 2) \mid$ are observed.

The decision mechanism implemented using a Fuzzy logic processor operates reliably, avoiding the generation of false symptoms in sections A, C, and E. The fault symptoms are processed by the detection logic block which confirms the presence of an arcing after 7 successive fault pulses (the tripping signal is activated in sections $\mathrm{B}$ and $\mathrm{D}$ ). The tripping activation is done in the starting arcing fault time (first wave cycle equivalent to $20 \mathrm{~ms}$ ). 


\subsubsection{Sub-case 2}

In Fig. 13 the transient operating mode of the current using the first masking-type configuration (Fig. 4) for a halogen lamp, vacuum cleaner 2 and a drill is shown. The current registered in section A exhibits stationary behavior while the load connected in the line is only resistive $(R=80 \Omega)$. In section $\mathrm{B}$ the halogen lamp is started with a maximum power variation giving rise to a transient state which affects the Kalman filter dynamic. Consequently, residuals and $\left|\operatorname{dif} f^{3}(X 2)\right|$ coefficients with high amplitudes are generated. However, the Fuzzy logic thresholding prevents fault symptoms being generated and, as a result, false tripping by the detection logic block is not produced. In section $\mathrm{C}$ an arcing fault is produced when the current waveform reaches the stationary state. In this section Res have low amplitudes in comparison to $\left|\operatorname{diff}^{3}(X 2)\right|$ coefficients which have a sufficient number of high persistent amplitudes to identify an arcing fault. Finally, the arcing fault is confirmed and the tripping signal is sent after three wave cycles $(60 \mathrm{~ms})$.

In the case of current waves generated by vacuum cleaner 2 , the transient behavior presented in section $\mathrm{D}$ occurs when the household appliance is switched on. Further, in the whole of this section, Res have high amplitudes with transient behavior. In contrast, $\left|d i f f^{3}(X 2)\right|$ coefficients are only affected by the presence of an arcing fault produced in section E, presenting high amplitudes. The detection logic block sends a tripping signal after $20 \mathrm{~ms}$.

The transient current behavior produced by a drill is presented in the whole of section F. In section $G$ high amplitudes are observed for both Res and $\left|\operatorname{diff} f^{3}(X 2)\right|$ coefficients due to an arcing fault. The influence of the arcing fault in the two input parameters allows the thresholding logic to generate a good number of fault symptoms (high density). The detection logic block sends a tripping after a wave cycle (20 ms).

Fault symptoms in transient conditions under the first masking type configuration are effectively obtained in spite of the parallel $(R=80 \Omega)$ resistor which masks arcing faults.

\subsubsection{Sub-case 3}

In Fig. 14, the transient operating mode of the current using the second masking-type configuration is shown. The drill current is recorded, taking into consideration working conditions (variable speed drilling). Sections A and B show transient current behavior with a decreasing trend. In section $\mathrm{B}$, the drilling process induces a motor torque variation. However, a very slightly variation in the amplitude of the signal current is presented. Arcing faults generated at regular time intervals (sections $\mathrm{B}, \mathrm{D}, \mathrm{F}$ and $\mathrm{H}$ ) causes high amplitude values on $\left|\operatorname{diff}^{3}(X 2)\right|$ coefficients. In contrast, Res have high amplitudes in all sections (A, B, C, D, E, F, G and H) since they are not affected by an arcing fault. This phenomenon is favored by the masking configuration which produces a very weak distortion in current waves making them difficult to identify from healthy waves. Nonetheless, the thresholding logic generates sufficient number of fault symptoms. The detection logic block sends a tripping signal in each arcing fault event (after $80 \mathrm{~ms}$ ). An undesirable effect produced by an internal state on the timer causes the appearance of $t_{d 1}$ and $t_{d 2}$ which represent the remaining times where tripping signal remains active despite the arcing extinction. It is also important to note that a single false symptom not considered by the detection logic block is generated in section $\mathrm{G}$.

\subsubsection{Sub-case 4}

In Fig. 15, the transient operating mode of the current using the third masking-type configuration is shown. The current of vacuum cleaner 2 is registered when vacuuming the floor. In section $\mathrm{A}$, the masking configuration does not allow the transient effect on the current to be visualized (when vacuum cleaner 2 is switched on). However, transient conditions causes a transient variation of Res amplitude (green frame). The vacuuming action starts in section $\mathrm{B}$ and produces a motor torque variation. Consequently, the masking configuration and the working conditions produce distortion in the shape of current waves which cannot be completely tracked by the Kalman filter. As a result, Res remains with high amplitudes in all sections. Only High amplitudes of $\left|\operatorname{diff}^{3}(X 2)\right|$ coefficients are observed in sections B and D when a series arcing fault is produced. However, the masking configuration reduces the sensitivity of the thresholding logic and, as a consequence, the number of fault symptoms decreases. The detection logic block sends the tripping signal after $100 \mathrm{~ms}$.

\subsubsection{Sub-case 5}

The algorithm developed is able to reliably detect arcing fault events during transient loads conditions taking into account different masking-type configurations. However, it is also imperative to test the algorithm's robustness using an EMI filter.

The EMI filters reduce the parasitic noise present in electrical systems and are essentially used to avoid nuisance trips from conventional AFCI circuits. However, using these filters can significantly reduce the sensitivity of protection systems masking arcing faults. According to standard IEC 62606, EMI filter 1 presented in Fig. 5 was tested under an arcing fault event and different types of loads ( $47 \Omega$ resistor, a drill and vacuum cleaner 2 ). Then, $i_{\text {measured }}$ was processed by the algorithm thus developed in order to detect the presence of a fault. The results of fault detection are shown in Fig. 16. As can be seen, EMI filter 1 reduces the sensitivity of the thresholding logic with the least number of fault symptoms generated when using a resistor as load. Nevertheless, the detection logic block succeeds in sending a tripping signal when using a drill and vacuum cleaner 2 as loads; distortions in the current in the presence of arcing faults are more perceptible. This effect is favored by the combined effect produced by the switching process of inductive loads, as well as the length of the transmission line and capacitor values used in the filter implementation. These features intensify arcing faults making them easily detectable by the decision part.

\subsection{Case 3: Parallel disturbing appliance}

The configuration shown in Fig. 6, uses different disturbing household appliances (Table 1). Different tests were performed in order to evaluate the performance of the proposed algorithm. 


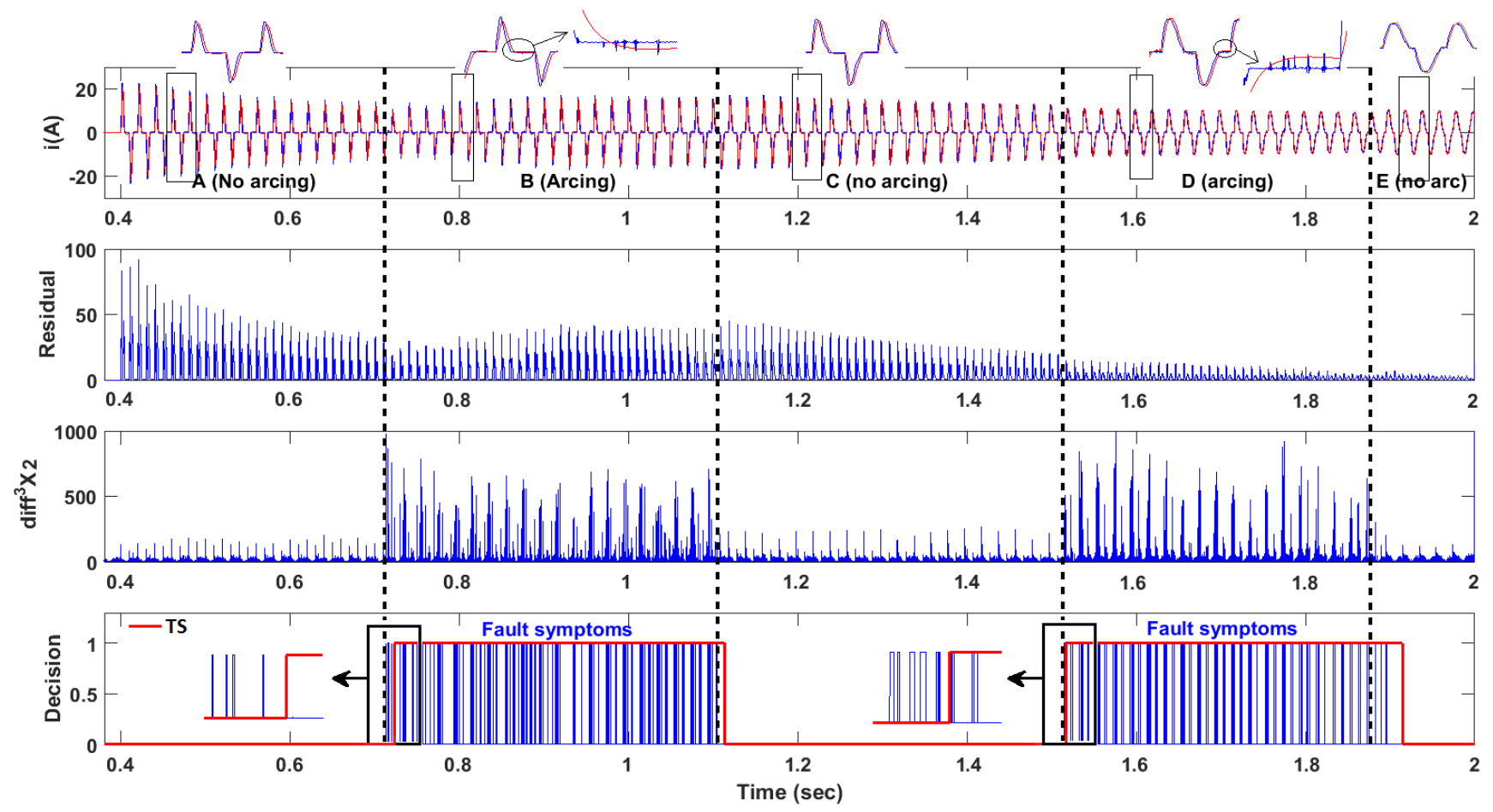

Figure 12: Sub-case 1: transient current of vacuum cleaner 2.
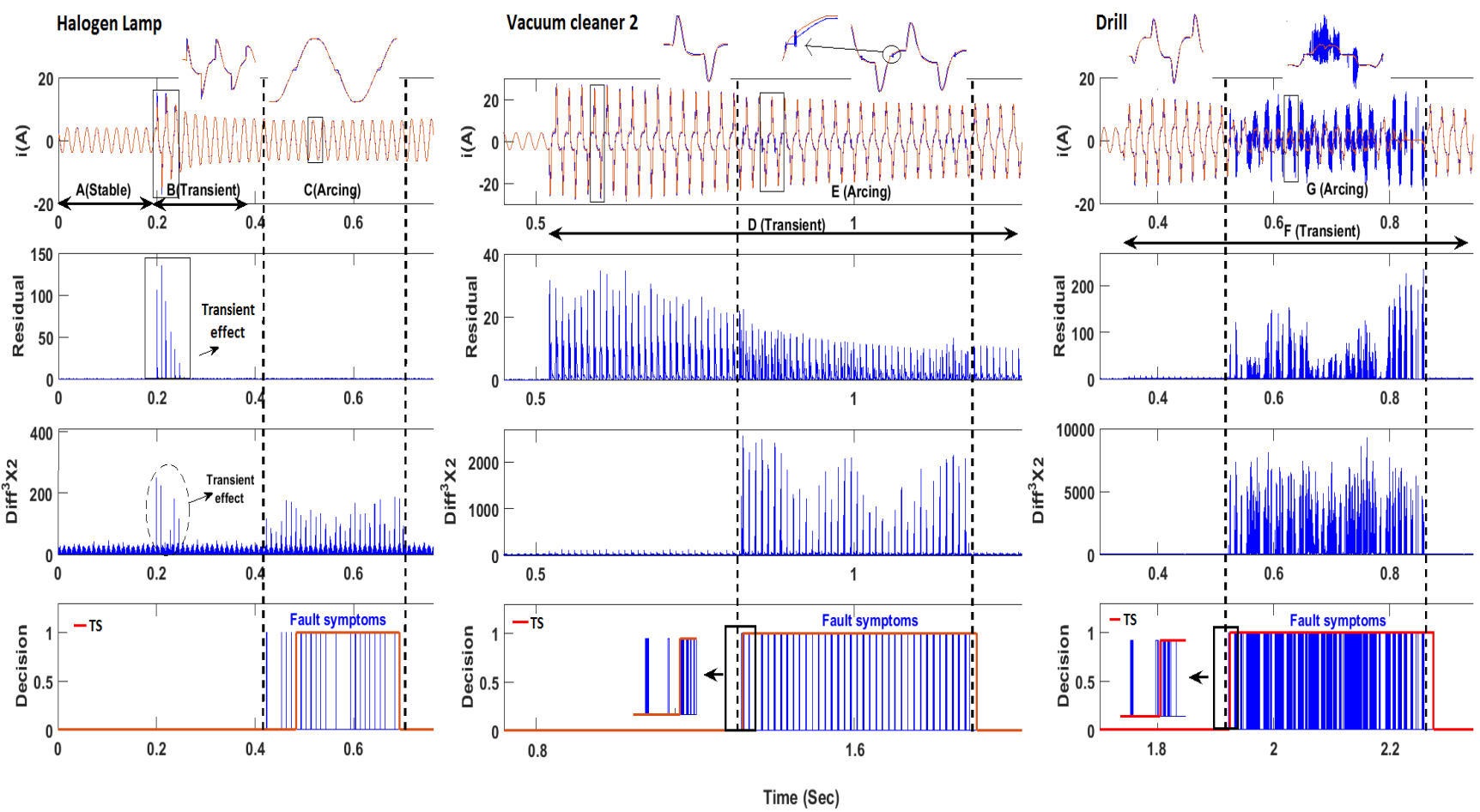

Figure 13: Sub-case 2: transient current (masking-type 1) of an halogen lamp, vacuum cleaner 2 and a drill.

The disturbing effects produced by a drill, vacuum cleaner 1 , a PC and a fluorescent lamp with ballast do not affect the decision block because the thresholding logic generates fault symptoms only when an arcing fault is produced.

However, the disturbing configuration, using a compressor as a perturbing load, introduces a large amount of noise in the electrical network. The kalman filter identifies the presence of an arcing fault but the decision block is affected. Consequently, the Fuzzy rules employed by the thresholding logic do not correspond to the operating conditions imposed by the perturba- 


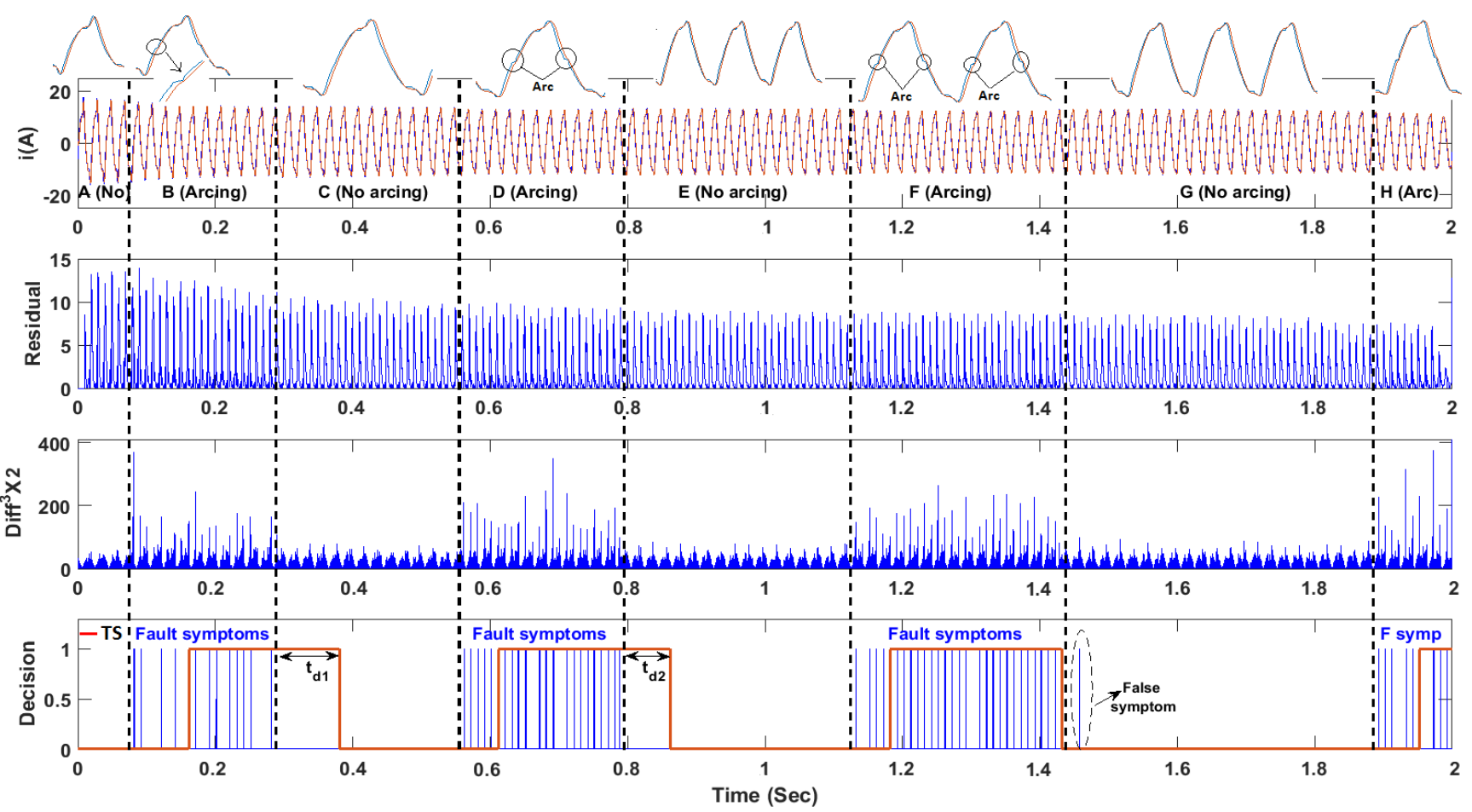

Figure 14: Sub-case 3: transient current (masking-type 2) of a drill.

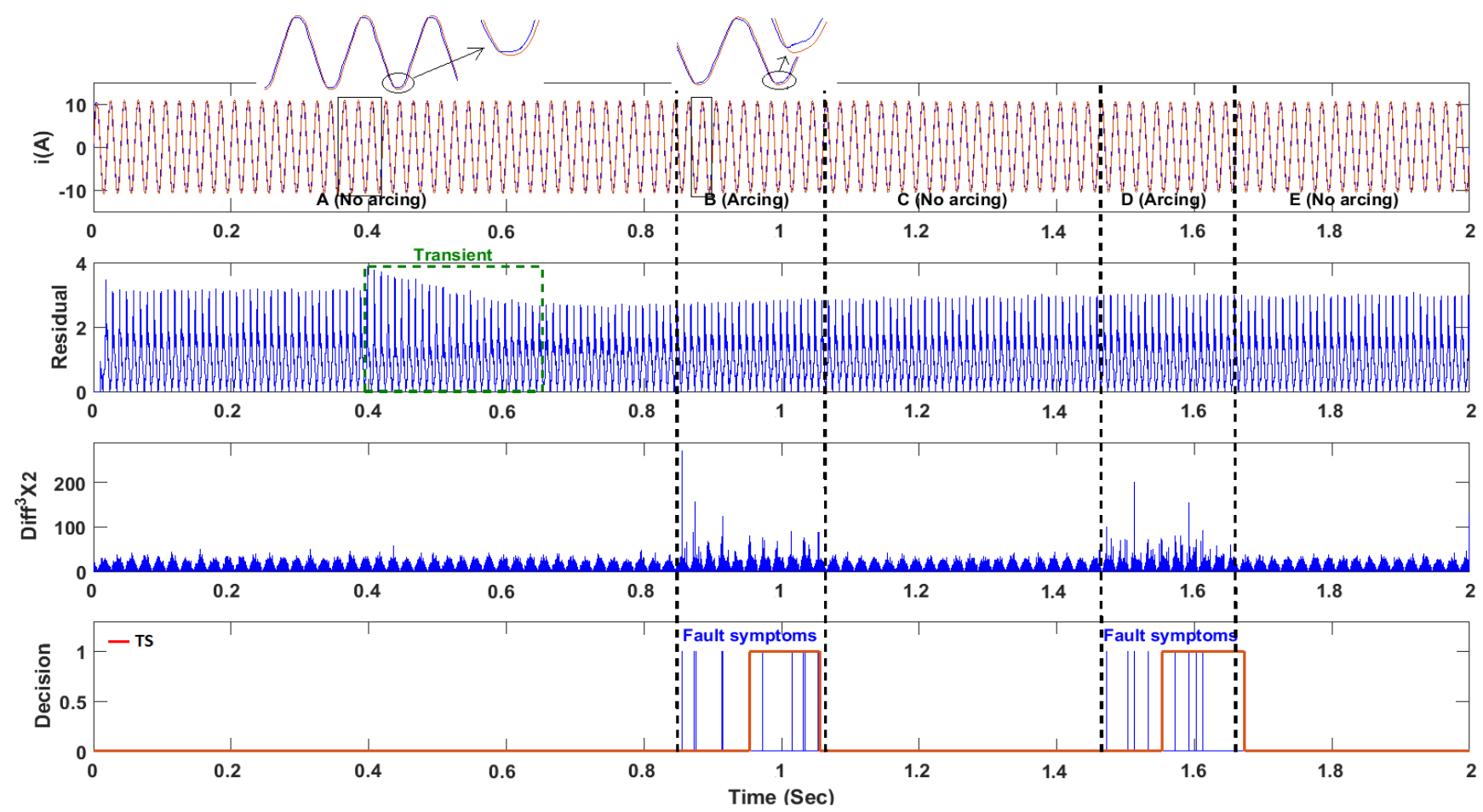

Figure 15: Sub-case 4: transient current (masking-type 3) of vacuum cleaner 2.

tion. These conditions produced by a high power disturbing load provide false tripping alarms in normal circuit operation. A simple solution to avoid false tripping consists in estimating the current flow of the disturbing appliance. If this current is high $(>50 \mathrm{~A})$ the algorithm then selects a static threshold fixed at 800 (dimensionless value).

Fig. 17 displays the current waveform and the tripping signal for vacuum cleaner 2 . The transient state is visualized in section A. The disturbing load is switched on in section B, generating a new transient condition. An arcing fault is then ignited 

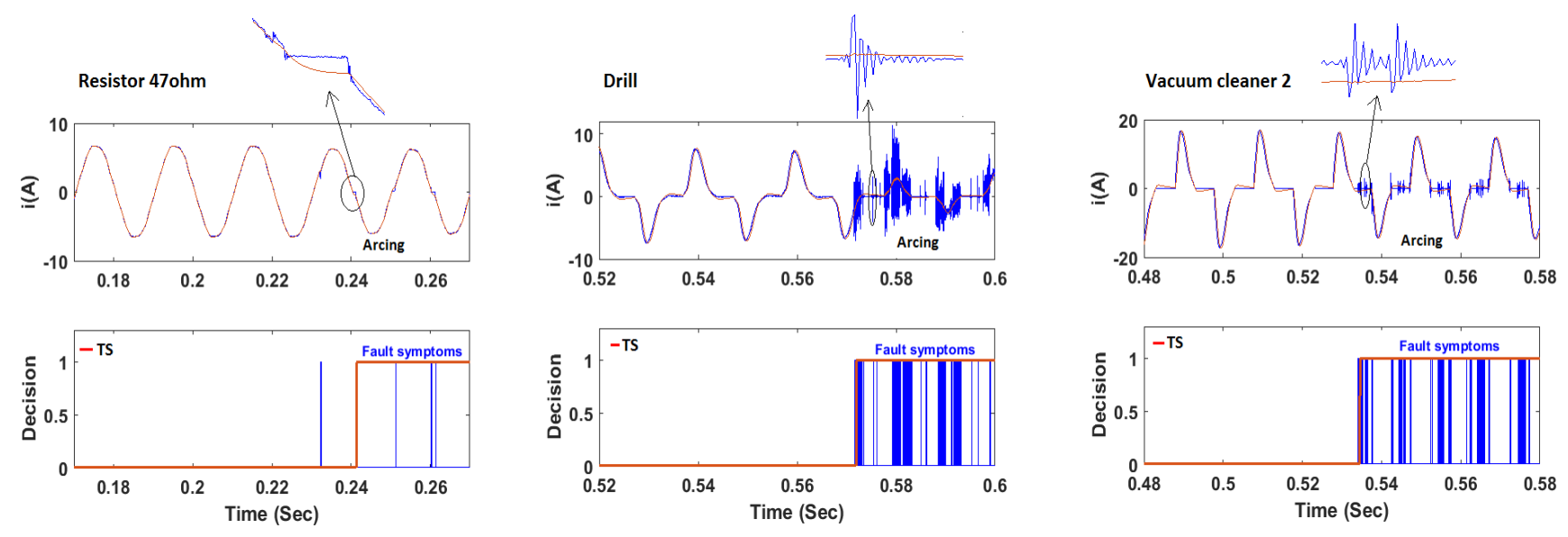

Figure 16: Sub-case 5: fault detection using an EMI filter.
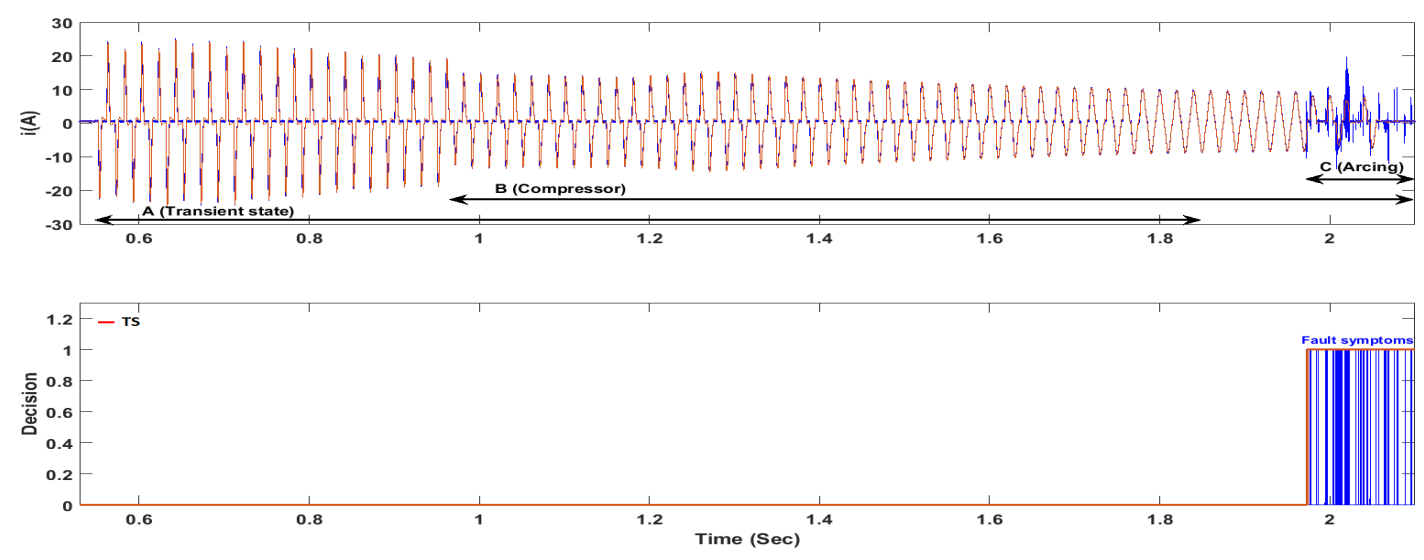

Figure 17: Transient current of the vacuum cleaner 2 (with disruptive compressor).
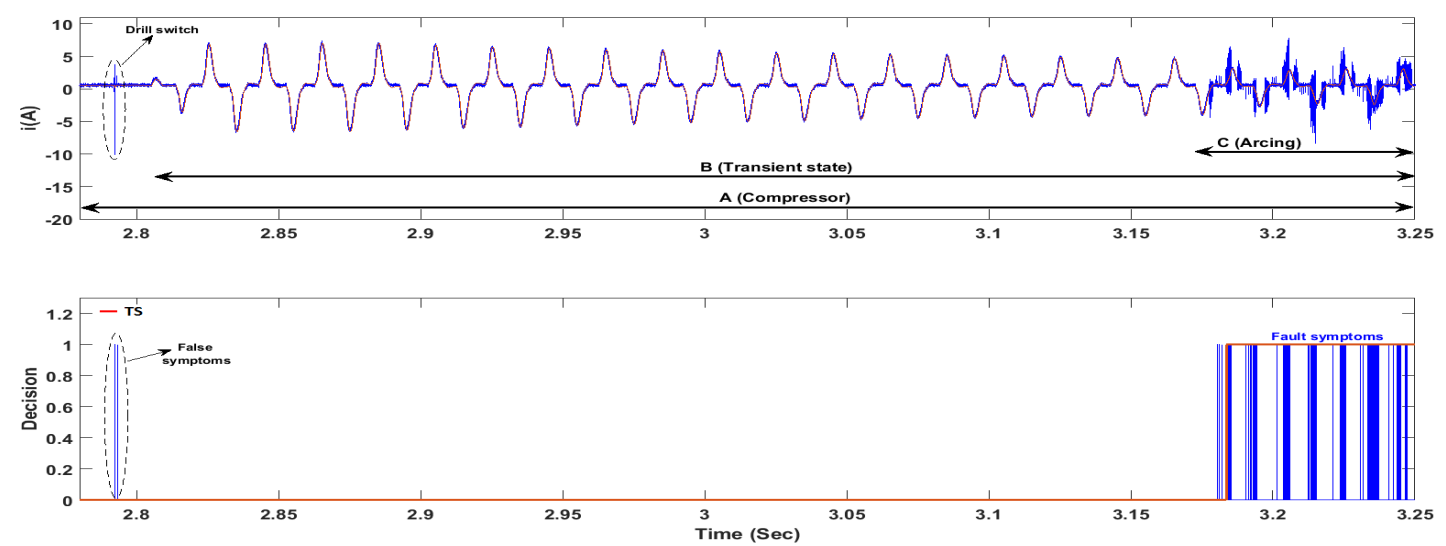

Figure 18: Transient current of a drill (with disruptive compressor).

in presence of a disturbing load (compressor). The static threshold generates fault symptoms which are processed by the logic detection block to send a tripping signal only in presence of an arcing fault at $t=1.975 \mathrm{~s}$.

Fig. 18 shows the current waveform and the tripping sig- nal for a drill. In this case the disturbing load is activated the whole time (Section A). The transient condition is also shown in section B. We notice the one-off presence of fault symptoms at $t=2.79 \mathrm{~s}$ which are not interpreted as an arc fault. On the other hand, in the case of a sustained arcing fault, the tripping 
signal is activated at $t=3.185 \mathrm{~s}$.

Table 4 summarizes the performances obtained by all the tests carried out in the laboratory.

Table 4: Summary of results.

\begin{tabular}{|c|c|c|c|c|c|}
\hline $\begin{array}{l}\text { Operation } \\
\text { Mode }\end{array}$ & \multicolumn{2}{|c|}{ Load Configuration } & $\begin{array}{l}\text { No. of } \\
\text { Tests }\end{array}$ & $\begin{array}{l}\text { Diagnosis } \\
\text { Accuracy } \\
(\%)\end{array}$ & $\begin{array}{l}\text { Average } \\
\text { Detection } \\
\text { Time (ms) }\end{array}$ \\
\hline \multirow{2}{*}{ Stationary } & \multicolumn{2}{|c|}{ Simple } & 20 & 100 & 60 \\
\hline & \multicolumn{2}{|c|}{ Parallel } & 4 & 100 & 40 \\
\hline \multirow{9}{*}{ Transient } & \multicolumn{2}{|c|}{ Simple } & 8 & 100 & 20 \\
\hline & \multicolumn{2}{|c|}{ Parallel } & 7 & 100 & 30 \\
\hline & \multicolumn{2}{|c|}{ Masking } & 18 & 100 & 60 \\
\hline & \multicolumn{2}{|c|}{ EMI filter } & 4 & 100 & 40 \\
\hline & Disturbing & Drill & 3 & 100 & 20 \\
\hline & \multirow[t]{4}{*}{ Load } & V. cleaner1 & 2 & 100 & 30 \\
\hline & & $\mathrm{PC}$ & 2 & 100 & 30 \\
\hline & & F. lamp & 2 & 100 & 35 \\
\hline & & Compressor & 4 & 75 & 40 \\
\hline
\end{tabular}

Table 5 presents a comparison of the proposed method with recently proposed approaches. From this comparison, it can be seen that the architecture of the proposed method based on a Kalman filter has the important advantage to detect arcing faults in the transient regime considering different maskingtype configurations (including EMI tests) on a larger number of loads (simple and combined). Also, the greatest advantage of the proposed method compared to proposed approaches [4, $6,7,9,14,25]$ is the using of an adaptive thresholding mechanism which can avoid efficiently unwanted trips in the process of fault detection without requiring complex training tasks as are used in approaches [27-29]. Moreover, concerning the time response, the proposed method gives satisfactory results and exceeds the requirements defined by the standard IEC 62606 (tripping time $=0.12 \mathrm{~s}$ for line current $=32 \mathrm{~A}$ ).

\section{Conclusion and future work}

In this paper, we present an original method for series arcing fault detection in a home electrical installation. The proposed algorithm is based on a Kalman filter (detection block) which operates at $100 \mathrm{kHz}$ and a Fuzzy logic processor (decision block). The generation of Res and $\left|\operatorname{diff}^{3}(X 2)\right|$ coefficients obtained essentially from a Kalman filter provide us with a first arcing fault detection approach. A second step allows us to select different fault symptoms and to confirm the arcing fault through the use of a decision part. The core of the decision part is composed by a Fuzzy logic processor which generates a threshold with different amplitude levels. The results obtained in this work shows that series arc fault can be successfully detected in presence of non-linear household appliances during the transient phase and also in steady-state regime. Furthermore, the performance of this algorithm is not affected by load variations, different masking load configurations and disturbing appliances which can generate false detections. We show that the adaptive threshold technique, implemented in this work causes significant reduction in false tripping by the detection logic block in comparison to a fixed threshold used by conventional approaches or architectures based on neural networks and SVM. Finally, our results show that the detection block confirms an arcing fault after counting seven fault pulses in a timing window of $\delta=60 \mathrm{~ms}$ experimentally determined. The results obtained in both stationary and transient operation mode satisfy the triggering time imposed by standards UL 1699 and IEC 62606 with a $230 \mathrm{~V}$ supply voltage. Future work aims to test loads under the influence of EMI perturbations (presented in a home electrical network) according to EN 61000-4-4, EN 61000-4-5 and EN 61000-4-11 standards. The implementation of the algorithm in an embedded electronic board is also considered.

\section{Acknowledgement}

This work was supported by the Peruvian National Program for Scholarships and Educational Loans (PRONABEC).

\section{References}

[1] Nedzad Hadziefendic, Miomir Kostic, and Zoran Radakovic. "Detection of series arcing in low-voltage electrical installations". In: European Transactions on Electrical Power 19.3 (2009), pp. 423-432.

[2] P. Muller, S. Tenbohlen, R. Maier, and M. Anheuser. "Characteristics of Series and Parallel Low Current Arc Faults in the Time and Frequency Domain". In: 2010 Proceedings of the 56th IEEE Holm Conference on Electrical Contacts. Oct. 2010, pp. 1-7.

[3] Dae-won Park, Il-kwon Kim, Su-yeon Choi, and Gyung-suk Kil. "Detection algorithm of series arc for electrical fire prediction”. In: 2008 International Conference on Condition Monitoring and Diagnosis. Apr. 2008, pp. 716-719.

[4] G. Artale, A. Cataliotti, V. Cosentino, D. Di Cara, S. Nuccio, and G. Tinè. "Arc Fault Detection Method Based on CZT Low-Frequency Harmonic Current Analysis". In: IEEE Transactions on Instrumentation and Measurement 66.5 (May 2017), pp. 888-896.

[5] D. C. Yu and S. H. Khan. "An adaptive high and low impedance fault detection method". In: IEEE Transactions on Power Delivery 9.4 (Oct. 1994), pp. 1812-1821.

[6] Slavisa Jovanovic, Abderrazak Chahid, Jinmi Lezama, and Patrick Schweitzer. "Shunt active power filter-based approach for arc fault detection”. In: Electric Power Systems Research 141 (2016), pp. 11-21.

[7] A. Mukherjee, A. Routray, and A. K. Samanta. "Method for Online Detection of Arcing in Low-Voltage Distribution Systems". In: IEEE Transactions on Power Delivery 32.3 (June 2017), pp. 1244-1252.

[8] C.J. Kim and B.D. Russell. "Analysis of distribution disturbances and arcing faults using the crest factor". In: Electric Power Systems Research 35.2 (1995), pp. 141-148.

[9] Jinmi Lezama, Patrick Schweitzer, Etienne Tisserand, Jean-Baptiste Humbert, Serge Weber, and Patrice Joyeux. "An embedded system for AC series arc detection by inter-period correlations of current". In: Electric Power Systems Research 129 (2015), pp. 227-234.

[10] Etienne Tisserand, Jinmi Lezama, Patrick Schweitzer, and Yves Berviller. "Series arcing detection by algebraic derivative of the current”. In: Electric Power Systems Research 119 (2015), pp. 91-99.

[11] H. Gu, F. Zhang, Z. Wang, Q. Ning, and S. Zhang. "Identification method for low-voltage Arc fault based on the loose combination of wavelet transformation and neural network". In: 2012 Power Engineering and Automation Conference. Sept. 2012, pp. 1-4.

[12] F. B. Costa. "Fault-Induced Transient Detection Based on Real-Time Analysis of the Wavelet Coefficient Energy". In: IEEE Transactions on Power Delivery 29.1 (Feb. 2014), pp. 140-153. 
Table 5: Comparison of the proposed method with recently proposed approaches.

\begin{tabular}{|c|c|c|c|c|c|c|c|c|c|c|}
\hline & \multicolumn{2}{|c|}{ Type of loads } & \multirow[b]{2}{*}{ Combined loads } & \multirow{2}{*}{$\begin{array}{c}\text { Transient } \\
\text { regime }\end{array}$} & \multicolumn{2}{|c|}{ Masking configuration } & \multirow{2}{*}{$\begin{array}{c}\text { EMI } \\
\text { test } \\
\text { filter }\end{array}$} & \multirow{2}{*}{$\begin{array}{c}\text { Detection } \\
\text { logic }\end{array}$} & \multirow{2}{*}{$\begin{array}{c}\text { Time } \\
\text { response }\end{array}$} & \multirow{2}{*}{$\begin{array}{l}\text { Electronic board } \\
\text { implementation }\end{array}$} \\
\hline & Linear loads & Nonlinear loads & & & $\begin{array}{c}\text { Stationary } \\
\text { regime }\end{array}$ & $\begin{array}{c}\text { Transient } \\
\text { regime }\end{array}$ & & & & \\
\hline [9] & 1 & 2 & Dimmer//R & No & No & No & No & FT & $10 \mathrm{~ms}$ & MBED LCP1768 \\
\hline [6] & 4 & 5 & $\begin{array}{c}\text { AC-DC power supply//R } \\
\text { Light bulb//R } \\
\text { Dimmer//R }\end{array}$ & No & $\begin{array}{l}\text { Yes } \\
\text { (MT 1) }\end{array}$ & No & No & FT & $20 \mathrm{~ms}$ & FPGA Stratix III \\
\hline [27] & 2 & 3 & Vacuum cleaner//R & No & $\begin{array}{c}\text { Yes } \\
\text { (MT 1) }\end{array}$ & No & No & ANN & $600 \mathrm{~ms}$ & No \\
\hline [4] & 2 & 5 & $\begin{array}{c}\text { Dimmer//Lamp } \\
\text { Fluorescent lamps//R }\end{array}$ & No & $\begin{array}{l}\text { Yes } \\
\text { (MT 1) }\end{array}$ & No & No & FT & $\begin{array}{l}80 \mathrm{~ms} \text { to } \\
400 \mathrm{~ms}\end{array}$ & No \\
\hline [29] & 1 & 4 & No & No & No & No & No & ANN & $500 \mathrm{~ms}$ & No \\
\hline [28] & 1 & 8 & No & Yes & No & No & No & ANN & NS & No \\
\hline [14] & 1 & 2 & No & No & No & No & No & FT & $100 \mathrm{~ms}$ & No \\
\hline [25] & 2 & 4 & $\mathrm{PC} / / \mathrm{PC}$ & No & No & No & No & FT & $160 \mathrm{~ms}$ & No \\
\hline [7] & 1 & 2 & $\begin{array}{c}\text { Dimmer//Light bulb } \\
\text { CPU1//CPU2//CPU3 } \\
\text { Dimmer//bulb//CPU1//CPU2//CPU3 }\end{array}$ & No & No & No & No & FT & NS & No \\
\hline $\begin{array}{c}\text { Proposed } \\
\text { method }\end{array}$ & 3 & 7 & $\begin{array}{c}\text { Halogen lamp// Halogen lamp } \\
\mathrm{PC} / / \mathrm{PC} \\
\text { Vacuum cleaner//R } \\
\text { Drill//R } \\
\end{array}$ & Yes & Yes & Yes & Yes & AT & $\begin{array}{c}20 \mathrm{~ms} \text { to } \\
200 \mathrm{~ms}\end{array}$ & No \\
\hline
\end{tabular}

[13] K. Koziy, B. Gou, and J. Aslakson. "A Low-Cost Power-Quality Meter With Series Arc-Fault Detection Capability for Smart Grid". In: IEEE Transactions on Power Delivery 28.3 (July 2013), pp. 1584-1591.

[14] Hong-Keun Ji, Guoming Wang, Woo-Hyun Kim, and Gyung-Suk Kil. "Optimal design of a band pass filter and an algorithm for series arc detection”. In: energies 11.992 (2018), pp. 1-13.

[15] W. Liu, X. Zhang, Y. Dong, and X. Huang. "Arc fault detection for AC SSPC based on Hilbert-Huang transform". In: IECON 2017 - 43rd Annual Conference of the IEEE Industrial Electronics Society. Oct. 2017, pp. 4104-4109.

[16] Pan Qi, Slavisa Jovanovic, Jinmi Lezama, and Patrick Schweitzer. "Discrete wavelet transform optimal parameters estimation for arc fault detection in low-voltage residential power networks". In: Electric Power Systems Research 143 (2017), pp. 130-139.

[17] R. Doraiswami and L. Cheded. "Kalman filter for parametric fault detection: an internal model principle-based approach". In: IET Control Theory Applications 6.5 (Mar. 2012), pp. 715-725.

[18] Qinghua Zhang. “Adaptive Kalman Filter for Actuator Fault Diagnosis”. In: IFAC-PapersOnLine 50.1 (2017). 20th IFAC World Congress, pp. 14272-14277.

[19] Zhentong Liu and Hongwen He. "Sensor fault detection and isolation for a lithium-ion battery pack in electric vehicles using adaptive extended Kalman filter". In: Applied Energy 185 (2017). Clean, Efficient and Affordable Energy for a Sustainable Future, pp. 2033-2044.

[20] Wei Gao, Jingchun Li, Guangtao Zhou, and Qian Li. "Adaptive Kalman Filtering with Recursive Noise Estimator for Integrated SINS/DVL Systems". In: Journal of Navigation 68.1 (2015), pp. 142-161.

[21] Xifeng Li, Yongle Xie, Dongjie Bi, and Yongcai Ao. "Kalman Filter Based Method for Fault Diagnosis of Analog Circuits". In: 2013.

[22] A. A. Girgis. "A New Kalman Filtering Based Digital Distance Relay". In: IEEE Transactions on Power Apparatus and Systems PER-2.9 (Sept. 1982), pp. 65-66.

[23] S. R. Samantaray, L. N. Tripathy, and P. K. Dash. "Combined EKF and SVM based High Impedance Fault detection in power distribution feeders". In: 2009 International Conference on Power Systems. Dec. 2009, pp. 1-6.

[24] T. Ghanbari. "Kalman filter based incipient fault detection method for underground cables". In: IET Generation, Transmission Distribution 9.14 (2015), pp. 1988-1997.

[25] Shiwen Zhang, Feng Zhang, Peng Liu, and Zhengzhi Han. "Identification of low voltage AC series arc faults by using kalman filtering algorithm". In: Elektronika Ir Elecktrotechnika 20.5 (2014), pp. 51-56.
[26] L. Karlöf, T.A. Ølgård, F. Godtliebsen, M. Kaczmarska, and H. Fischer. "Statistical techniques to select detection thresholds for peak signals in ice-core data". In: Journal of Glaciology 51.175 (2005), pp. 655-662.

[27] Yu-Wei Liu, Chi-Jui Wu, and Yi-Chieh Wang. "Detection of serial arc fault on low-voltage indoor power lines by using radial basis function neural network". In: International Journal of Electrical Power $\mathcal{G}$ Energy Systems 83 (2016), pp. 149-157.

[28] Kai Yang, Rencheng Zhang, Shouhong Chen, Fujiang Zhang, Jianhong Yang, and Xingbin Zhang. "Series Arc Fault Detection Algorithm Based on Autoregressive Bispectrum Analysis". In: Algorithms 8.4 (2015), pp. 929-950.

[29] Y. Wang, F. Zhang, and S. Zhang. "A New Methodology for Identifying Arc Fault by Sparse Representation and Neural Network". In: IEEE Transactions on Instrumentation and Measurement (2018), pp. 1-12.

[30] Z. Yi and A. H. Etemadi. "Fault Detection for Photovoltaic Systems Based on Multi-Resolution Signal Decomposition and Fuzzy Inference Systems". In: IEEE Transactions on Smart Grid 8.3 (May 2017), pp. 1274-1283.

[31] S. Dhar and P. K. Dash. "Differential current-based fault protection with adaptive threshold for multiple PV-based DC microgrid". In: IET Renewable Power Generation 11.6 (2017), pp. 778-790.

[32] B. Grichting, J. Goette, and M. Jacomet. "Cascaded fuzzy logic based arc fault detection in photovoltaic applications". In: 2015 International Conference on Clean Electrical Power (ICCEP). June 2015, pp. 178183.

[33] H. Sneider and P. M. Frank. "Observer-based supervision and fault detection in robots using nonlinear and fuzzy logic residual evaluation". In: IEEE Transactions on Control Systems Technology 4.3 (May 1996), pp. 274-282.

[34] Matthew B. Rhudy. A Kalman Filtering Tutorial for Undergraduate Students. Mar. 2018.

[35] K. Phipps, T. Cooke, D. Dorr, and P. Keebler. "Frequency phenomenon and algorithms for arc detection". In: 2010 IEEE International Symposium on Electromagnetic Compatibility. July 2010, pp. 183-188.

[36] Y. C. Kang, S. H. Ok, and S. H. Kang. "A CT saturation detection algorithm”. In: 2003 IEEE Power Engineering Society General Meeting (IEEE Cat. No.03CH37491). Vol. 1. July 2003, pp. 33-33.

[37] Byung Eun Lee, Jinsik Lee, Sung Ho Won, Byongjun Lee, Peter A. Crossley, and Yong Cheol Kang. "Saturation Detection-Based Blocking Scheme for Transformer Differential Protection”. In: Energies 7.7 (2014), pp. 4571-4587. 